\title{
Inhibiting Lithium Dendrites in Lithium Metal Batteries
}

ISSN: 2578-0255

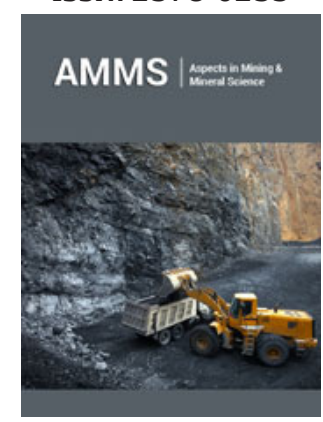

*Corresponding author: Yuping $\mathrm{Wu}$, State Key Laboratory of Materials-oriented Chemical Engineering \& School of Energy Science and Engineering, Nanjing Tech University, Nanjing 211816, China

Submission: 眥 January 30, 2020

Published: 眥April 13, 2020

Volume 4 - Issue 5

How to cite this article: Xiaosong Xiong, Qi Zhou, Yusong Zhu, Yuping Wu, et al. Inhibiting Lithium Dendrites in Lithium Metal Batteries. Aspects Min Miner Sci. 4(5). AMMS.000596. 2020.

DOI: 10.31031/AMMS.2020.04.000596

Copyright@ Yuping Wu, This article is distributed under the terms of the Creative Commons Attribution 4.0 International License, which permits unrestricted use and redistribution provided that the original author and source are credited.

\author{
Xiaosong Xiong ${ }^{1}$, Qi Zhou ${ }^{1}$, Yusong Zhu ${ }^{1}$, Yuhui Chen ${ }^{1}$, Lijun Fu ${ }^{1}$, Lili Liu ${ }^{1}$, Nengfei \\ $\mathrm{Yu}^{1}$, Yuping $\mathrm{Wu}^{1 *}$ and Teunis van $\mathrm{Re}^{2}$
}

${ }^{1}$ State Key Laboratory of Materials-oriented Chemical Engineering \& School of Energy Science and Engineering, Nanjing Tech University, China

${ }^{2}$ Department of Chemistry, University of Venda, South Africa

\begin{abstract}
Characterized by high theoretical specific capacity $(3860 \mathrm{mAh} / \mathrm{g})$ and the lowest reduction potential $(-3.04 \mathrm{~V})$, the lithium metal anode has received much attention in the continuous pursuit of highperformance batteries. However, the problems of uncontrollable lithium dendrite growth and the high chemical reactivity of lithium, which result in low coulombic efficiency and short cycle life of lithium metal batteries, have remained unsolved for decades. Even worse, the presence of lithium dendrites poses serious risks to battery safety. In recent years, much work has been conducted on the issue of lithium dendrites. In this review, we summarize the latest basic strategies for solving the lithium dendrite problem, including the choice of liquid electrolytes, the application of solid/gel electrolytes, modification on separator, and tailored surface and scaffold for lithium metal anode. In addition, challenges and prospects of lithium metal anodes are discussed.
\end{abstract}

Keywords: lithium dendrites; Graphite; Batteries; Materials

\section{Introduction}

As an important part of portable electronics and new energy vehicles, batteries have become an important factor restricting their rapid development. Of various available rechargeable batteries, lithium-ion batteries have been commercialized for large-scale applications due to their high specific capacity, long cycle life and good safety performance [1]. After continuous improvement, the current specific energy density of commercial lithium-ion batteries is approaching its theoretical value but still cannot meet the growing needs for higher energy densities. Normally, graphite is employed as an active intercalation anode material; during the charge process, the $\mathrm{Li}^{+}$ions intercalate into the graphite, while the opposite occurs during the discharge process. Due to the limited space between graphite layers, its theoretical specific capacity is restricted to $372 \mathrm{mAh} / \mathrm{g}$ [2]. As a result, an anode system with a higher energy density has to be found urgently. Studies on lithium metal anodes started in the 1970s. When lithium metal is used as the anode, its mechanism is based on deposition or stripping of $\mathrm{Li}^{+}$ ions, with its theoretical specific capacity reaching $3860 \mathrm{mAh} / \mathrm{g}$. Furthermore, because it has the lowest known reduction potential $\left(-3.04 \mathrm{~V}\right.$ vs S.H.E.) and low density $\left(0.59 \mathrm{~g} / \mathrm{cm}^{3}\right)$ [3], the theoretical specific capacity of the battery can be increased greatly, so that lithium metal is one of the most promising anode materials. However, the formation of lithium dendrites has been a major problem for lithium metal anodes for a long time. Suffering from the uncontrollable growth of lithium dendrites on the lithium metal anode, lithium metal batteries exhibit low coulombic efficiency and short cycle life. Even worse, lithium dendrites give rise to safety hazards because they cause short-circuits $[4,5]$.

Much research has been conducted to understand the formation of lithium dendrites. To date, dendrite formation is understood as follows: due to the high chemical reactivity of lithium, the lithium metal reacts with the electrolyte to form a solid-electrolyte interface (SEI) layer, which can protect the lithium metal from side reactions after assembly of the lithium metal battery. During the charging process, the $\mathrm{Li}^{+}$ions tend to plate in the form of an uneven and loose lithium deposition layer, so that the volume of the anode changes dramatically during the charge and discharge processes, inducing cracks in the fragile SEI. The exposed fresh lithium reacts with the electrolyte to repair the SEI, and the repeating process will lead 
to a great loss of active lithium and electrolyte. Meanwhile, $\mathrm{Li}^{+}$ions accumulate at the tip region of the deposition where there is a stronger electric field during electrochemical deposition, leading to rapid growth of dendritic lithium. On the one hand, the electrical contact between lithium dendrites and substrate is broken to form "dead lithium," shortening the cycle life significantly. On the other hand, it is quite probable that dendrites will pierce through the separator and cause an internal short-circuit, posing a safety hazard [6-8]. Obviously, formation of a uniform and robust SEI is crucial to alleviation of the growth of lithium dendrites. In this review, we summarize some promising methods to inhibit lithium dendrite formation, including the aspects of electrolyte, separator, and anode. Furthermore, challenges and further directions for research are also discussed.

\section{Choice of liquid electrolytes}
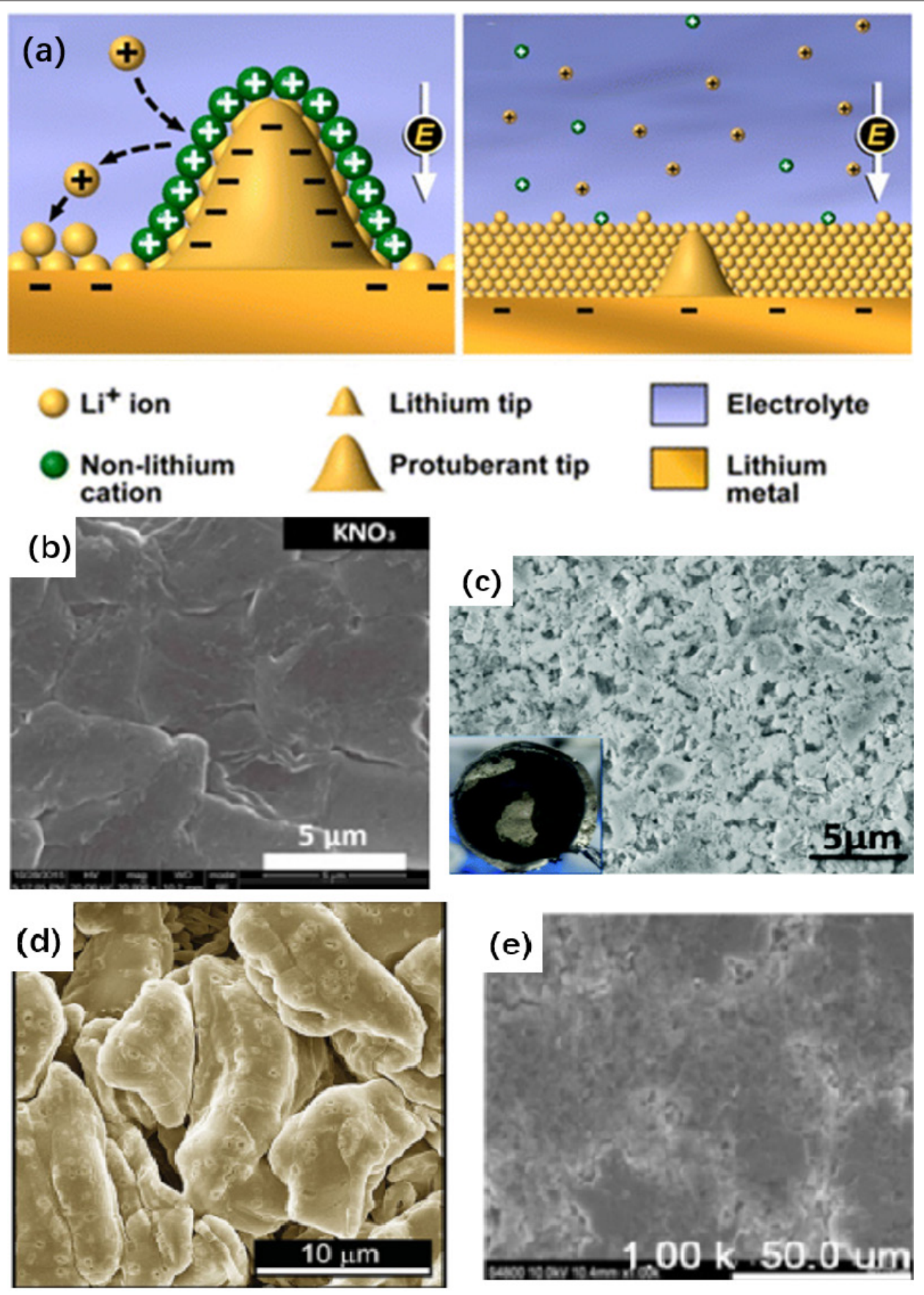

Figure 1: (a) Illustration of Li deposition process based on the self-healing electrostatic shield mechanism [16],

(b) SEM images of deposited Li metal on $\mathrm{Li}-\mathrm{Cu}$ cells with $\mathrm{KNO}_{3}$ additive [17],

(c) SEM images of the metallic Li electrodes cycled in 5M LiFSI/TMP electrolyte [18],

(d) The top view SEM images after first cycle lithium plating [19], and

(e) surface morphologies of the Li-metal anode in Li/Cu cells with $2 \mathrm{M} \mathrm{LiFSI+2M} \mathrm{LiFTFSI} \mathrm{electrolyte} \mathrm{after} 100$ cycles at $0.5 \mathrm{~mA} / \mathrm{cm} 2$ [21]. Reprinted with permission from Ref. [16-19,21]. Copyright American Chemical Society. 
Side reactions between lithium metal and liquid electrolyte cannot be avoided because of the high chemical reactivity of lithium, but the as-formed SEI is crucial because it does not only protect the lithium metal from side reactions but also influences the deposition morphology of lithium. $\mathrm{Li}^{+}$ions will deposit preferentially on heterogeneous surfaces of the electrode with defects, causing an uneven lithium deposition and then triggering the growth of lithium dendrites. An ideal SEI should therefore exhibit high ionic conductivity, homogeneous composition and high elastic strength to accommodate the volume changes of the electrode. Because the solvents, lithium salts and additives have a significant influence on the formation of the SEI, it is feasible and convenient to regulate the SEI by optimizing electrolyte components [9]. Compared with carbonate solvents, ether solvents present better compatibility with the Li electrode and are commonly used as electrolyte solvent in lithium metal batteries; for example, 1,3-dioxolane (DOL), 1,2-dimethoxyethane (DME) and tetra(ethylene glycol) dimethyl ether (TEGDME) are prone to form oligomers in SEI layers [10]. Mostly $\mathrm{LiClO}_{4}, \mathrm{LiCF}_{3} \mathrm{SO}_{3}$ (LiTFS) and $\mathrm{LiC}_{2} \mathrm{~F}_{6} \mathrm{NO}_{4} \mathrm{~S}_{2}$ (lithium bis (trifluoromethanesulfonyl) imide, LiTFSI) serve as lithium salts [11]. Electrolyte additives also have an important effect on SEI formation, and can help to form a robust SEI by participating in the SEI-forming reactions. Fluoroethylene carbonate (FEC) [12], vinylene carbonate (VC) [13] and $\mathrm{LiNO}_{3}$ [14] are representatives of traditional additives that react preferentially with lithium to form a superior SEI film on the anode and thus reduce the consumption of electrolyte. Some metal cations with low reduction potentials, such as $\mathrm{Na}^{+}, \mathrm{Cs}^{+}$and $\mathrm{Rb}^{+}[15,16]$ are also very effective electrolyte additives (Figure 1a). When lithium deposition starts to become uneven, the charge accumulation at the tip regions will attract these cations to gather at the protrusions, forming an electrostatic protective shield, impeding the local accumulation of lithium ions, and alleviating the growth of dendrites. Added $\mathrm{KNO}_{3}$ has been shown to perform better than $\mathrm{LiNO}_{3}$. For example, in the case of $0.1 \mathrm{M} \mathrm{KNO}_{3}$ in 1M LiTFSI (DME:DOL=1:1 v/v) electrolyte, a Li-S battery exhibits an average discharge capacity of $687 \mathrm{mAh} / \mathrm{g}$, higher than the $637 \mathrm{mAh} / \mathrm{g}$ obtained with $0.1 \mathrm{M} \mathrm{LiNO}_{3}$ additive. This enhancement can be attributed to the electrostatic shielding effect of $\mathrm{K}^{+}$, while the lithium deposit is more uniform due to the inhibition of lithium dendrite growth (Figure 1b) [17]. When electrons transfer faster than $\mathrm{Li}^{+}$ions during the deposition process, there will be a concentration gradient near the surface of the anode, and lithium dendrites are expected to form easily at this time. High electrolyte concentrations $(>2 \mathrm{M})$ has been shown to alleviate this problem $[18,19]$. High lithium ion concentrations can lower the concentration gradient near the surface of the anode, so that the generation of space charge is suppressed (Figure 1c). The solvation of lithium ions is decreased, accelerating mass transfer, which is beneficial for the uniform deposition of lithium. By employing $4 \mathrm{M}$ lithium bis(fluorosulfonyl)imide (LiFSI)-DME electrolyte, improved coulombic efficiency and long cycle life are ensured. In a Li/Cu halfcell using this electrolyte, an average coulombic efficiency of $98.4 \%$ over more than 1000 cycles at a current density of $4 \mathrm{~mA} / \mathrm{cm}^{2}$ was obtained. The control half-cell using $1 \mathrm{M} \mathrm{LiFSI-DME} \mathrm{electrolyte} \mathrm{failed}$ in just a few cycles with a highly fluctuating coulombic efficiency. The observation of nodule-like lithium deposition morphology by
SEM showed that lithium dendrite formation was suppressed in 4M LiFSI-DME electrolyte (Figure 1d) [20]. A dendrite-free Li metal anode was also attained using 7M LiFSI in FEC (Figure 1e) [21] and 2M LiFSI+2M LiTFSI in DME [22].

An ionic liquid (IL) electrolyte is a type of low temperature molten salt, which is composed entirely of ions. In lithium metal batteries, IL electrolytes can be used to tune the SEI and promote the homogeneous deposition of lithium [23,24]. However, due to their high viscosity, which will decrease the ionic conductivity, it is usually necessary to use liquid solvents for dilution. The room temperature ionic conductivity of a diluted and solvated ionic liquid composed of tetraethylene glycol dimethyl ether solvent (G4) and LiFSI diluted to $50 \mathrm{vol} \%$ with DOL reached $4.82 \times 10^{-3} \mathrm{~S} \cdot \mathrm{cm}^{-1}$, and the $\mathrm{Li} / \mathrm{Cu}$ half-cell using this optimized electrolyte exhibited excellent coulombic efficiency of greater than $99.98 \%$ at $5 \mathrm{~mA} / \mathrm{cm}^{2}$ [25]. Other solvents such as DME [26] and TEGDME [27,28] are also effective in increasing the ionic conductivity of ionic liquids and improving the characteristics of the lithium metal anode.

\section{Use of solid or gel electrolytes}

Characterized by excellent electrochemical and thermal stability, superior mechanical properties and relatively high lithium ionic conductivity, solid electrolytes are attracting more and more attention as a promising method to suppress the growth of lithium dendrites and obtain a safer lithium metal anode [29]. Solid electrolytes can be generally classified into inorganic and polymer electrolytes. Inorganic solid electrolytes include sulfides, oxides and phosphates such as $\mathrm{Li}_{10} \mathrm{GeP}_{2} \mathrm{~S}_{12}$ [30], $\mathrm{Li}_{3} \mathrm{PS}_{4}[31], \mathrm{Li}_{1.3} \mathrm{Al}_{0.3} \mathrm{Ti}_{1.7}\left(\mathrm{PO}_{4}\right)_{3}$ [32] and $\mathrm{Li}_{2} \mathrm{P}_{5} \mathrm{O}_{6} \mathrm{~N}_{5}$ [33], while the main components of polymer solid electrolytes are polymers such as PAN, PEO, PMMA and PVDF [34]. However, solid electrolytes have some shortcomings, such as insufficient ionic conductivity, large interfacial resistance and poor compatibility with lithium metal, which hinder their direct use in lithium metal batteries [35]; some modification is needed. Doping is a common method to increase ionic conductivity. For instance, $\mathrm{Li}_{1.3} \mathrm{Al}_{0.3} \mathrm{Ti}_{1.7}\left(\mathrm{PO}_{4}\right)_{3}$ results from doping of $\mathrm{LiTi}_{2}\left(\mathrm{PO}_{4}\right)_{3}$ solid electrolyte with $\mathrm{Al}$, which greatly increases the ionic conductivity to an order of $10^{-3} \mathrm{~S} / \mathrm{cm}$ due to the increased lithium content and the densification of the solid electrolyte [32]. Similarly, the interfacial compatibility and stability between solid electrolyte and lithium metal anode can be improved by adding $\mathrm{Li}_{3} \mathrm{PO}_{4}$ and $\mathrm{LiF}$ $[36,37]$. Combining the advantages of the high ionic conductivity of a liquid electrolyte and the high safety of a solid electrolyte, gel polymer electrolytes (GPE) are an effective way to suppress lithium dendrites for safer lithium metal batteries. An example is the pentaerythritol tetraacrylate (PETEA)-based GPE. Its ionic conductivity is $1.13 \times 10^{-2} \mathrm{~S} / \mathrm{cm}$ at room temperature. Because of the reduced lithium dendrite formation and the strong immobilization of polysulfides in the presence of PETEA, the capacity retention ratio of the Li-S battery is $81.9 \%$ after 400 cycles at $0.5{ }^{\circ} \mathrm{C}$. From the SEM images of the lithium deposition after 50 cycles, the electrode retains relatively smooth morphology [28]. However, higher mechanical stability is necessary for effective shape conservation. Hence, a great deal of modifications of polymer gel electrolytes have been carried out, such as adding nano-fillers ( $\mathrm{ZnO}, \mathrm{MgO}$, 
$\mathrm{Al}_{2} \mathrm{O}_{3}, \mathrm{ZrO}_{2}$ ) and compounding with plasticizers (polyethylene, polystyrene) [38-41]. Construction of multilayer solid electrolytes is another promising solution to inhibit Li dendrite formation. The
Li-S battery applying LLCZN and PMMA bilayer solid electrolyte had an initial coulombic efficiency of $99.8 \%$, maintaining $99 \%$ in subsequent cycles at $0.2 \mathrm{~mA} / \mathrm{cm}^{2}$ (Figures $2 \mathrm{a} \& 2 \mathrm{~b}$ ) [42].

\section{Modified separators}
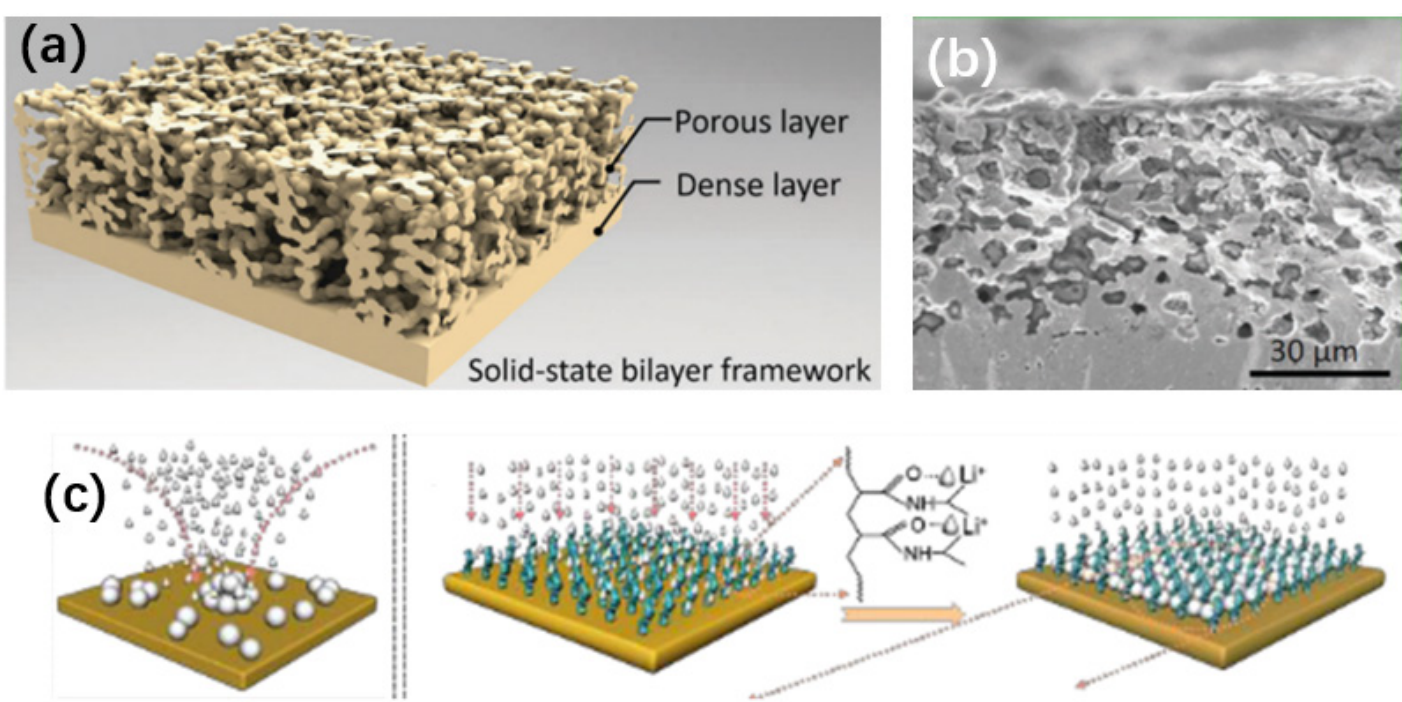

(d)

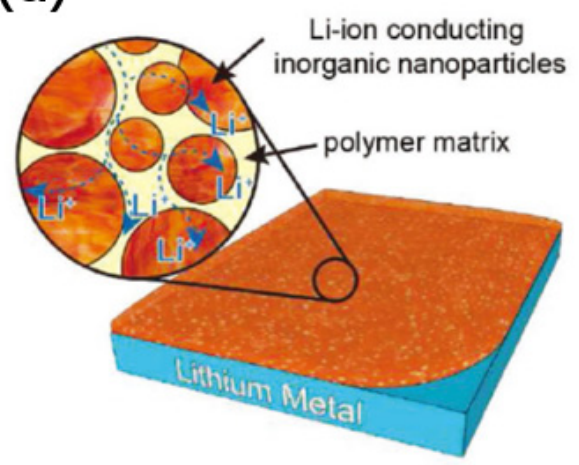

Li-ion conducting artificial SEI
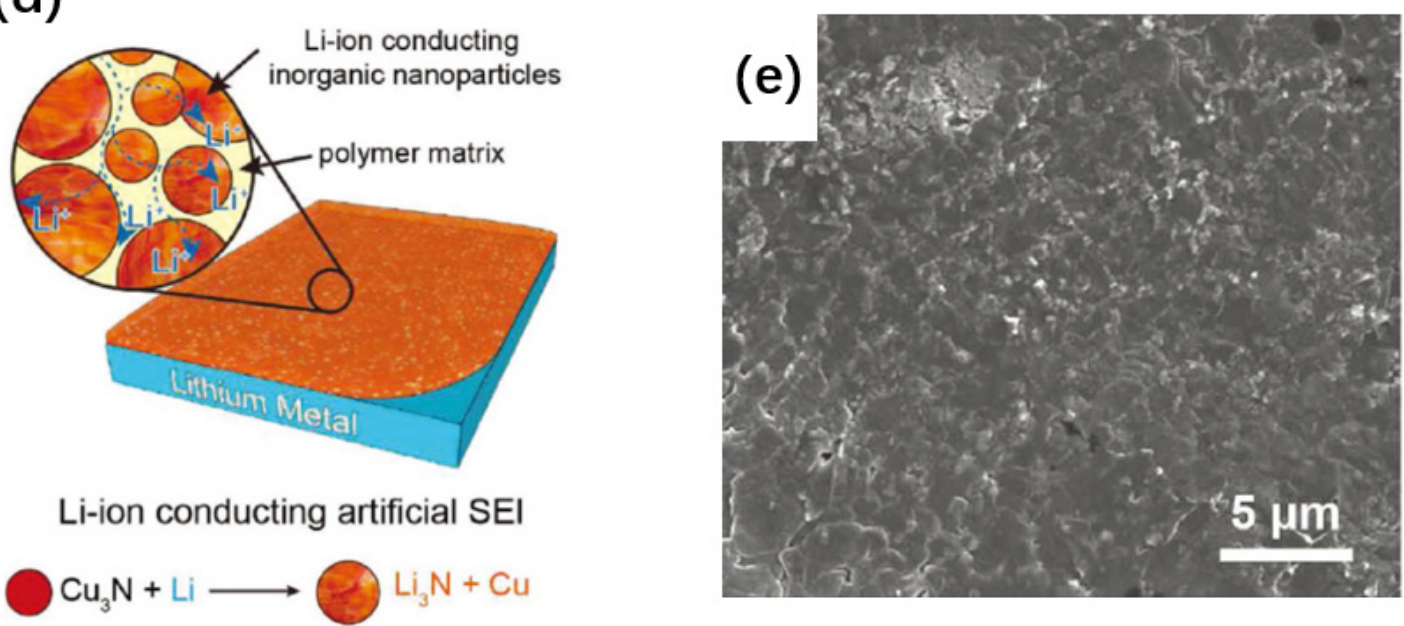

Figure 2: $(a-b)$ Schematic of the garnet bilayer framework and cross-section SEM image of the bilayer sulfur cathode [42],

(c) schematic illustration of Li nucleation on the bare $\mathrm{Cu}$ foil and PNIPAM grafted $\mathrm{Cu}$ substrates [52],

(d) schematic illustration of the $\mathrm{Cu}_{3} \mathrm{~N}+\mathrm{SBR}$ composite artificial SEI [53], and

(e) top-view SEM images after five galvanostatic plating/stripping cycles on $\mathrm{Cu}_{3} \mathrm{~N}+\mathrm{SBR}$ artificial SEI protected Cu

foil [53]. Reprinted with permission from Ref. [41,52,53]. Copyright of RSC Publishing and John Wiley and Sons.

The separator plays an important role in isolating the anode and cathode and providing a path for ion transport. Due to the close contact between the anode and the separator in an assembled cell, in addition to the basic physical barrier function, constructing modified layers on the separator could add the functions of tuning lithium deposition and inhibiting the growth of dendrites to the separator. Several types of materials can be used to modify separators, including carbon materials [43-45], polymeric materials [46], inorganic materials [47-49] and composites. Most of the carbon materials such as porous graphene [43], super-P [44] and acetylene black [45] are coated on the separator surface to inhibit the growth of lithium dendrites. However, a new strategy has been proposed to transform lithium dendrites into dense lithium deposits by modifying the commercial separator with functionalized nanocarbon (FNC). Para-benzenesulfonic acid groups on the FNC surface can immobilize some $\mathrm{Li}^{+}$ions during the initial cycle; in the subsequent cycles, dendrites will grow simultaneously on both the lithium metal anode and FNC coating. Once they make contact, the growth direction of the dendrites is changed, resulting in a dendrite-free uniform lithium deposition layer on the lithium metal surface. As shown in Figures $2 a \& 2 b$, the surface of the lithium metal anode is covered by a dense lithium deposit without obvious lithium dendrites. In sharp contrast, a great amount of lithium dendrites is formed on the control anode. From the perspective of 
electrochemical performance, $\mathrm{Li} / \mathrm{LiFePO}_{4}$ coin cells with modified separators can retain more than $80 \%$ of their initial capacity after 800 cycles at $1 \mathrm{C}$ in $1 \mathrm{M}$ LiTFSI (DOL:DME=1:1 v/v) [50]. The modified layer on the separator can also improve the interaction between the electrolyte and the separator to obtain better ionic conductivity and a higher lithium ion transference number, which will ameliorate the lithium ion deposition conditions and thereby suppress the growth of lithium dendrites and improve the performance of lithium metal batteries. For example, a polydopamine/octaammonium POSS (PDA/POSS) composite coating was applied on a PE polymer separator by mussel-inspired surface chemistry, which results in an increase in lithium ionic conductivity from 0.36 to $0.45 \mathrm{mS} /$ $\mathrm{cm}$, while the $\mathrm{Li}^{+}$transference number increases from 0.37 to 0.47 . As a result, $\mathrm{Li} / \mathrm{LiCoO}_{2}$ cells using a modified separator in $1 \mathrm{M} \mathrm{LiPF}_{6}$ (EC:EMC:DMC=1:1:1 v/v/v) show a discharge capacity retention of $83.4 \%$ after 200 cycles at $0.5 \mathrm{C}$, compared with $78.0 \%$ for the control batteries with pristine PE separator [51].

\section{Tailoring surface and scaffold of lithium metal}

There is no doubt that direct modification of lithium metal anodes is the most effective strategy to limit lithium dendrite formation [52,53]. Currently, research is mainly focused on surface modification and 3D composite matrices. It is hard to achieve precise regulation of the SEI during the charge/discharge process, and inhomogeneity of components and morphology is unavoidable. Therefore, ex-situ artificial coating of the lithium metal anode is regarded as a facile and effective means to guide the uniform lithium deposition and suppress the volume change of Li metal anode during cycling. The protective layer(s) on the surface of lithium metal must have high ionic conductivity, good stability and mechanical strength. By fine-tuning the preparation process, carbon materials such as carbon nanofiber (CNF) [54], multiwalled carbon nanotubes [55], ladder-like carbon nanoarrays [56] and nitrogen-doped amorphous carbon (a-CNx) films [57] were proven to be effective in inhibiting the growth of lithium dendrites. By electrospinning and magnetron sputtering techniques, a highly flexible semi-tubular carbon film was produced. This modified layer is highly insulated and has a densely interconnected semi-tube network structure, which helps to inhibit detrimental side reaction of the Li metal, and accommodates the huge volumetric change during the charge/discharge processes. Hence, the probability of lithium dendrite growth is greatly reduced. For $\mathrm{Li} / \mathrm{Cu}$ cells using a modified anode in 1M LiTFSI (DOL:DME=1:1, v/v) electrolyte with $1.0 \mathrm{wt} \% \mathrm{LiNO}_{3}$, the coulombic efficiency was increased to $99.2 \%$ at $0.5 \mathrm{~mA} / \mathrm{cm}^{2}$ within 125 cycles [58].

Due to the highly controllable functional groups on polymers and excellent wettability with the electrolyte, tailoring the lithium metal interface with flexible polymer coatings is another important direction for dendrite-free lithium metal anodes. To prevent the growth of lithium dendrites from the initial process, a 3D oxidized polyacrylonitrile (PAN) nanofiber polymer coating was prepared by co-electrospinning (Figure 3a). The polar functional group on the polymer provides a homogeneous lithium ion flux and prevents the uneven deposition of lithium; it also contributes to adsorption of a large amount of electrolyte, improving the affinity of the electrode for electrolyte. Furthermore, a 3D scaffold can better accommodate the volume changes of the deposited layer, which is conducive to uniform deposit morphology without dendrites (Figure 3b). The modified electrode was evaluated in a $\mathrm{Li} / \mathrm{Cu}$ cell with 1M LiTFSI (DOL:DME=1:1, v/v) and $2 \mathrm{wt} \% \mathrm{LiNO}_{3}$ as electrolyte. Cells with control electrodes had lower coulombic efficiencies and shorter cycle life, with the coulombic efficiency dropping to $70 \%$ after 60 cycles at a current density of $3 \mathrm{~mA} / \mathrm{cm}^{2}$. In contrast, cells with modified electrodes had an increased coulombic efficiency of $97.4 \%$ for 120 cycles [59]. Application of lithium alloys such as Li-Mg [59], Li-Al (Figure 2c) [60,61], and Li-Sn [62] is also a very effective method to protect lithium metal anodes. $\mathrm{Cu}_{3} \mathrm{~N}$ nanoparticles will form a fast ion conductor, $\mathrm{Li}_{3} \mathrm{~N}$, immediately when in contact with lithium metal. Fast lithium ion conductivity facilitates the rapid transport of lithium ions and ensures a uniform lithium ion flux near the electrode surface and inhibits the growth of lithium dendrites. A coating prepared by combining $\mathrm{Cu}_{3} \mathrm{~N}$ nanoparticles and styrene butadiene rubber (SBR) adds good synergistic flexibility (Figure 2d), so that a dendrite-free lithium metal anode is obtained (Figure 2e). In Li/Cu cells, the $\mathrm{Cu}_{3} \mathrm{~N}+\mathrm{SBR}$ protects $\mathrm{Cu}$ to deliver a relatively stable coulombic efficiency over 100 cycles, while the coulombic efficiency of pristine $\mathrm{Cu}$ fell below $70 \%$ in only 50 cycles at $1 \mathrm{~mA} /$ $\mathrm{cm}^{2}$ in $1 \mathrm{M} \mathrm{LiPF}_{6}$ (EC:DEC=1:1, v/v) electrolyte with $10 \mathrm{wt} \%$ FEC additive [53]. To alleviate the problem of local charge accumulation originating from uneven deposition and slow ion transport near the surface of the anode, 3D composite matrices have been proposed. On the one hand, the large specific surface area reduces the effective current density of the electrode, facilitates the uniform deposition and stripping of lithium, limits the lithium to a certain space, and greatly alleviates the drastic changes in the volume of the electrode. On the other hand, the loss of active lithium due to side reactions between electrolyte and metallic $\mathrm{Li}$ is reduced to some extent.

Reduced graphene oxide could bind with lithium strongly because of its good affinity with lithium, which guarantees the homogeneity of the layered Li-rGO composite electrode prepared by thermal infusion. Electrochemical tests showed that the Li-rGO electrode shows very small voltage hysteresis compared to the bare $\mathrm{Li}$ anode and the lithium stripping curve indicates that the composite anode can deliver a specific capacity of 3390mAh/g [63]. Highly cross-linked polymeric substrates also have 3D mesh frames and abundant pore channels where the $\mathrm{Li}^{+}$ions can be transported rapidly. Also, the method of electrospinning ensures that the matrix has no reactive activity and will not decompose during the cycling. A porous substrate was made from polyimide (PI), a chemically stable polymer with high mechanical strength, by electrospinning. A lithiophilic layer ( $\mathrm{ZnO}$ ) was then applied to solve the problem induced by the high interfacial tension of lithium on polyimide. After infusing molten lithium metal into the matrix, a composite Li anode was obtained (Figures 3c \& 3d). The electrochemical tests showed that the composite anode can be cycled stably for more than 100 cycles at a high current density of $5 \mathrm{~mA} / \mathrm{cm}^{2}$ in $1 \mathrm{M}$ $\mathrm{LiPF}_{6}(\mathrm{EC}: \mathrm{DEC}=1: 1, \mathrm{v} / \mathrm{v}$ ) [64]. In another direction, copper is well 
regarded as an anode current collector, and in most cases, copper foil sheets are used as the anode current collector. The 3D structure of the electrode can reduce the local current density to form a uniform lithium deposit without dendrites and to inhibit the volume change of the lithium metal. For example, a 3D Cu/Li composite anode displayed a coulombic efficiency of $93.8 \%$ after 100 cycles at $0.5 \mathrm{~mA} / \mathrm{cm}^{2}$, higher than the ordinary anode which exhibited only $30.9 \%$ efficiency after 70 cycles [65]. A 3D structure can also be formed on the surface of common copper foil by simple chemical methods. Firstly, immersing a $\mathrm{Cu}$ foil in ammonia solution to form a layer of $\mathrm{Cu}(\mathrm{OH})_{2}$ on the surface, then dehydrating, and reducing the $\mathrm{Cu}$ foil, a $\mathrm{Cu}$ foil with 3D porous surface is prepared (Figure 3e). At a current density of $0.5 \mathrm{~mA} / \mathrm{cm}^{2}$, the modified anode had a coulombic efficiency of $97 \%$ after 50 cycles in 1M LiTFSI (DOL:DME=1:1, v/v), which is much better than that of the unmodified $\mathrm{Cu}$ foil [66].

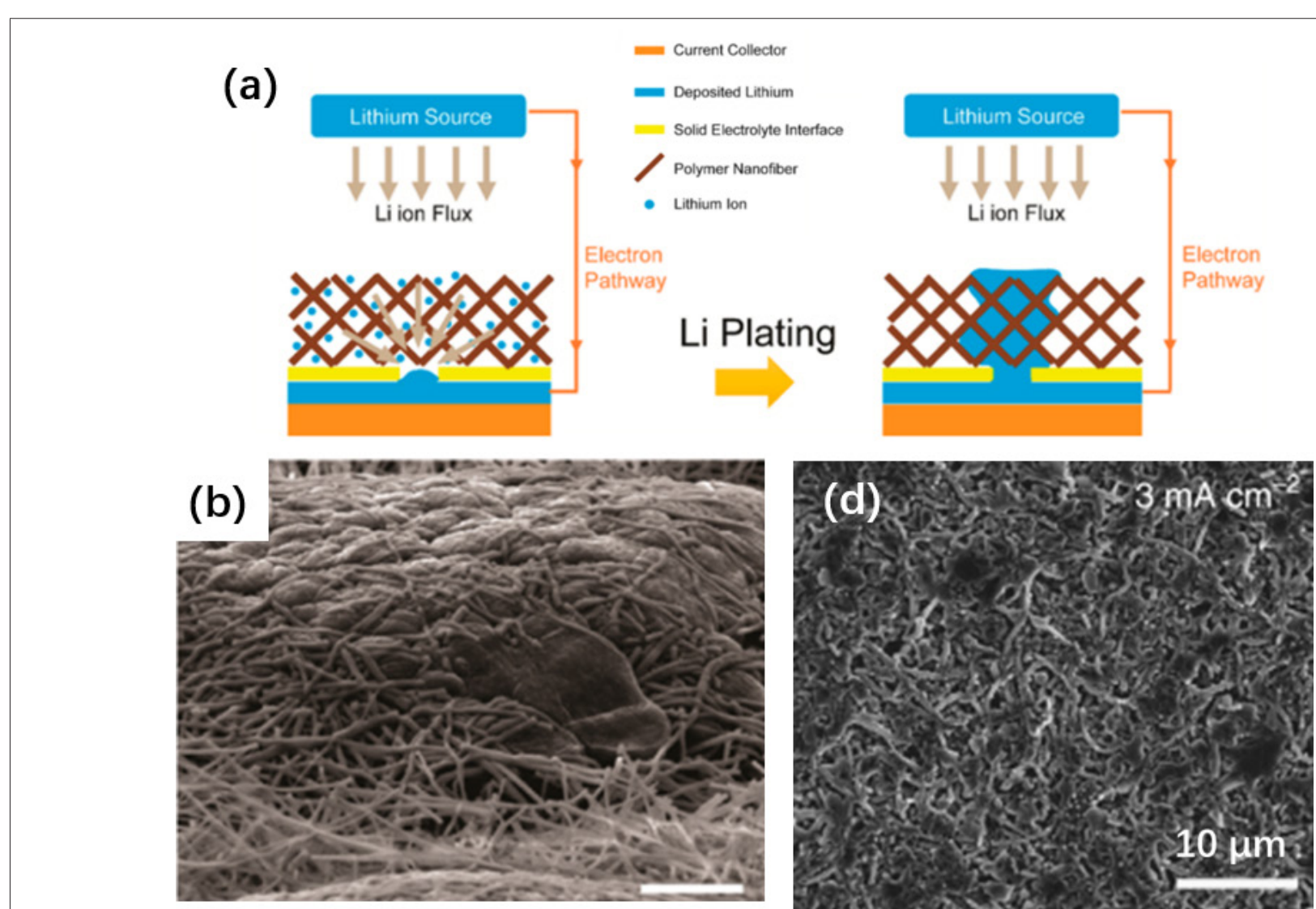

(c)
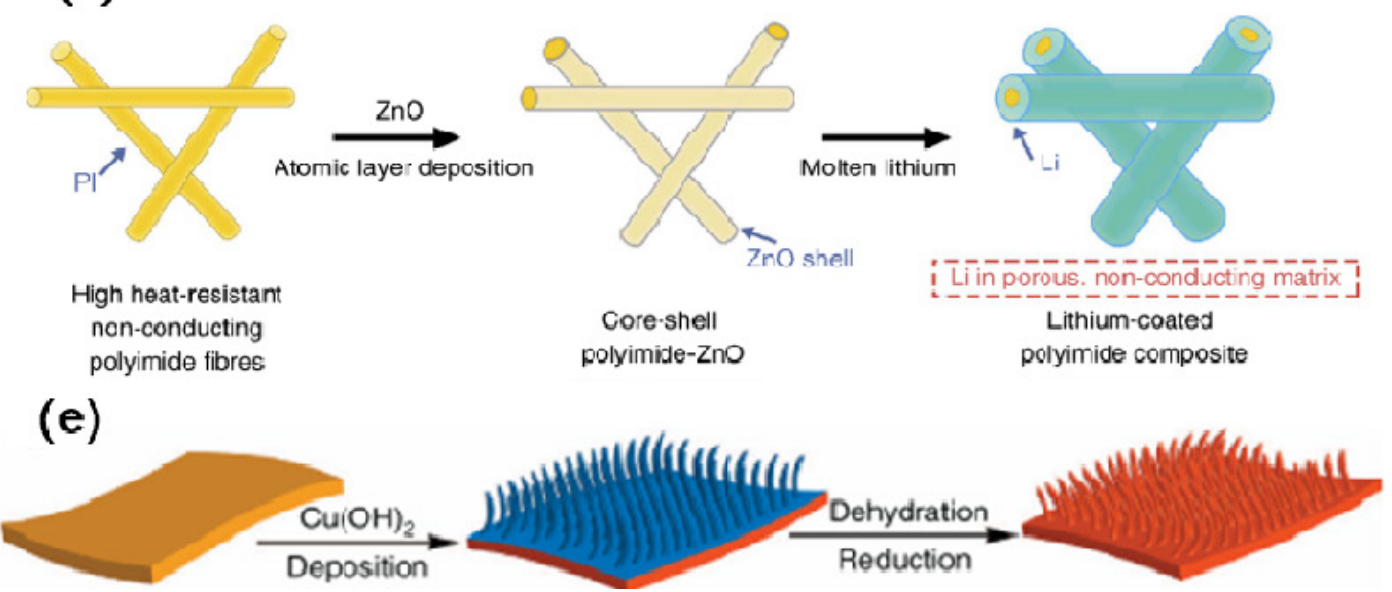

Figure 3: (a) Schematic of the polymer nanofiber modified layer [58],

(b) tilted-view SEM images of Li deposition on Cu-OxPA [58],

(c) top-view SEM images of the Li-coated polyimide (PI) matrix after 10 cycles of stripping/plating in EC/DEC at a current density of $3 \mathrm{~mA} / \mathrm{cm}^{2}$ [64],

(d) electrospun PI is coated with a layer of $\mathrm{ZnO}$ by ALD to form core-shell PI-ZnO [64], and

(e) schematic presentation of the procedures to prepare a 3D porous Cu foil from a planar Cu foil [66]. Reprinted with permission from Ref. $[58,64,66]$. Copyright ACS and Nature Springer. 


\section{Conclusion}

Safe lithium metal anodes have been an objective of research since the 1970s. However, the uncontrollable growth of lithium dendrites and low coulombic efficiency during the charge/ discharge processes still result in unsatisfactory practical performance of Li metal batteries. In this review, we discussed the formation and growth of lithium dendrites and the latest research on lithium metal anodes are also introduced: optimizing the composition of liquid electrolytes, applying solid/gel electrolytes, coating separators with functional layers, modifying the surface of lithium, and providing a host for lithium deposition. Although various improvement methods have been proposed, there still remain many challenges to producing a high-performance lithium metal anode. The formation of lithium dendrites is related to many factors, so that the improvement strategy should be comprehensive to meet the practical needs. Owing to the highly controllable functional groups available on polymers, excellent wettability with the electrolyte and superior flexibility, various polymer materials are widely used in lithium metal batteries as a component of the modified layer on an electrode or solid/gel electrolyte. We believe that combining the unique advantages of polymeric materials with other multi-functional components is a promising strategy in improving lithium metal anodes. Our understanding of the growth of lithium dendrites is still inadequate, and the established models are also not perfect to guide further improvement. As technology advances, more advanced simulation, testing and characterization technologies are being developed in the research on lithium metal anodes, which will accelerate the development of Li metal batteries. There are good prospects for practical introduction of Li metal batteries in the near future.

\section{Acknowledgement}

The authors gratefully acknowledge the financial support from the National Key R \& D Program of China (2018YFB0104300), National Natural Science Foundation Committee of China (Distinguished Youth Scientists Project of 51425301, U1601214, 21374021, 51573013, 51773092 and 51772147), State Key Lab Research Foundation (ZK201805, ZK201717), and Jiangsu Distinguished Professorship Program (2016).

\section{References}

1. Lu L, Han X, Li J, Hua J, Ouyang M (2013) A review on the key issues for lithium-ion battery management in electric vehicles. Journal of Power Sources 226: 272-288.

2. De las Casas C, Li w (2012) A review of application of carbon nanotubes for lithium ion battery anode material. Journal of Power Sources 208: 74-85.

3. Lin D, Liu Y, Cui Y (2017) Reviving the lithium metal anode for highenergy batteries. Nat Nanotechnol 12(3): 194-206.

4. Monroe C, Newman J (2003) Dendrite growth in lithium/polymer systems-A propagation model for liquid electrolytes under galvanostatic conditions. Journal of the Electrochemical Society 150(10): A1377-A1384.

5. Liu B, Zhang JG, Xu W (2018) Advancing lithium metal batteries. Joule 2(5): 833-845.
6. Harry KJ, Hallinan DT, Parkinson DY, MacDowell AA, Balsara NP (2014) Detection of subsurface structures underneath dendrites formed on cycled lithium metal electrodes. Nat Mater 13(1): 69-73.

7. Bieker G, Winter M, Bieker P (2015) Electrochemical in situ investigations of SEI and dendrite formation on the lithium metal anode. Phys Chem Chem Phys 17(14): 8670-8679.

8. Park MS, Ma SB, Lee DJ, Im D, Doo SG, et al. (2014) A highly reversible lithium metal anode. Sci Rep 4(1): 3815.

9. Yin YX, Xin S, Guo YG, Wan LJ (2013) Lithium-sulfur batteries: electrochemistry, materials, and prospects. Angew Chem Int Ed Engl 52(50): 13186-13200.

10. Scheers J, Fantini S, Johansson P (2014) A review of electrolytes for lithium-sulphur batteries. Journal of Power Sources 255: 204-218.

11. Hagos TT, Thirumalraj B, Huang CJ, Abrha LH, Hagos TM, et al. (2019) Locally concentrated $\mathrm{LiPF}_{6}$ in a carbonate-based electrolyte with fluoroethylene carbonate as a diluent for anode-free lithium metal batteries. ACS Appl Mater Interfaces 11(10): 9955-9963.

12. Brown ZL, Jurng S, Lucht BL (2017) Investigation of the lithium solid electrolyte interphase in vinylene carbonate electrolytes using $\mathrm{Cu}|| \mathrm{LiFePO}_{4}$ Cells. Journal of The Electrochemical Society 164(9): A2186-A2189.

13. Liang Zhang, Min Ling, Jun Feng, Liqiang Mai, Gao Liu, et al. (2018) The synergetic interaction between $\mathrm{LiNO}_{3}$ and lithium polysulfides for suppressing shuttle effect of lithium-sulfur batteries. Energy Storage Materials 11: 24-29.

14.Zhang Y, Qian J, Xu W, Russell SM,Chen X, et al. (2014) Dendrite-free lithium deposition with self-aligned nanorod structure. Nano letters 14(12): 6889-6896.

15. Ding F, Xu W, Graff GL, Zhang J, Sushko ML, et al. (2013) Dendrite-free lithium deposition via self-healing electrostatic shield mechanism. J Am Chem Soc 135(11): 4450-4456.

16. Jia W, Fan C, Wang L, Wang Q Zhou M, et al. (2016) Extremely accessible potassium nitrate (KNO3) as the highly efficient electrolyte additive in lithium battery. Acs Applied Materials \& Interfaces 8(24): 15399-15405.

17. Camacho Forero LE, Smith TW, Balbuena PB (2017) Effects of high and low salt concentration in electrolytes at lithium-metal anode surfaces. The Journal of Physical Chemistry C 121(1): 182-194.

18. Pengcheng S, Hao Zheng, Xin Liang, Yi Sun, Sheng Cheng, et al. (2018) A highly concentrated phosphate-based electrolyte for high-safety rechargeable lithium batteries. Chemical communications 54: 44534456.

19. Qian J, Adam BD, Zheng J, Xu W, Welsey A, et al. (2016) Anode-free rechargeable lithium metal batteries. Advanced Functional Materials 26(39): 7094-7102.

20. Suo L, Xue W, Gobet M, Greenbaum SG, Wang C, et al. (2018) Fluorinedonating electrolytes enable highly reversible 5-V-class $\mathrm{Li}$ metal batteries. Proceedings of the National Academy of Sciences 115(6): 1156-1161.

21. Ma Q, Fang Z, Liu P, Ma J, Qi X, et al. (2016) Improved cycling stability of lithium-metal anode with concentrated electrolytes based on lithium (fluorosulfonyl)(trifluoromethanesulfonyl) imide. ChemElectro Chem 3(4): 531-536.

22. Li NW, Yin YX, Li JY, Zhang CH, Guo YG (2017) Passivation of lithium metal anode via hybrid ionic liquid electrolyte toward stable li plating/ stripping. Adv Sci (Weinh) 4(2): 1600400.

23. Ding MS, Koch SL, Passerini S (2017) The effect of 1-pentylamine as solid electrolyte interphase precursor on lithium metal anodes. Electrochimica Acta 240: 408-414. 
24. Wang H, Matsui M, Kuwata H, Sonoki H, Matsuda Y, et al. (2017) A reversible dendrite-free high-areal-capacity lithium metal electrode. Nature Communications 8(1).

25. Yang Y, Fang M, Song Y, Zhou Y, Zhan H (2017) N-methoxyethyl-Nmethylpyrrolidinium bis (trifluoromethanesulfonyl) imide ionic liquid based hybrid electrolyte for lithium sulfur batteries. Electrochimica Acta 256: $37-43$

26. Wu F, Qizhen Zhu, Renjie Chen, Nan Chen, Yan Chen (2015) A Safe electrolyte with counterbalance between the ionic liquid and tris(ethylene glycol)dimethyl ether for high performance lithium-sulfur batteries. Electrochimica Acta 184: 356-363.

27. Liu M, Dong Zhou, Yan Bing He, Yongzhu Fu, Xianying Qin, et al. (2016) Novel gel polymer electrolyte for high-performance lithium-sulfur batteries. Nano Energy 22: 278-289.

28. Rawlence M, Garbayo I, Buecheler S, Rupp JL (2016) On the chemical stability of post-lithiated garnet Al-stabilized Li7La3Zr2012 solid state electrolyte thin films. Nanoscale 8(31): 14746-14753.

29. Yao X, Ning Huang, Fudong Han, Qiang Zhang, Hongli Wan, et al. (2017) High-performance all-solid-state lithium-sulfur batteries enabled by amorphous sulfur-coated reduced graphene oxide cathodes. Advanced Energy Materials 7(17).

30. Han F, Jie Yue, Xiulin Fan, Tao Gao, Chao Luo, et al. (2016) Highperformance all-solid-state lithium-sulfur battery enabled by a mixedconductive $\mathrm{Li}_{2} \mathrm{~S}$ nanocomposite. Nano Letters 16(7): 4521-4527.

31. Monchak M, Hupfer T, Senyshyn A, Hans Boysen, Chernyshov D, et al. (2016) Lithium diffusion pathway in $\mathrm{Li}_{1.3} \mathrm{Al}_{0.3} \mathrm{Ti}_{1.7}\left(\mathrm{PO}_{4}\right)_{3}$ (LATP) Superionic Conductor. Inorg Chem 55(6): 2941-2945.

32. Meesala Y, Jena A, Chang H, Liu RS (2017) Recent advancements in Li-ion conductors for all-solid-state Li-ion batteries. Acs Energy Letters 2(12): 2734-2751.

33. Xiong, X., Wenqi Yan, Chaolin You, Yusong Zhu, Yuhui Chen, et al. (2019) Methods to improve lithium metal anode for Li-S batteries. Frontiers in Chemistry.

34. Lin D, Liu W, Liu Y, Lee HR, Hsu PC, et al. (2016) High ionic conductivity of composite solid polymer electrolyte via in situ synthesis of monodispersed $\mathrm{SiO}_{2}$ nanospheres in poly(ethylene oxide). Nano Lett 16(1): 459-465.

35. Xu B, Li W, Duan H, Wang $\mathrm{H}$, Guo Y, et al. (2017) $\mathrm{Li}_{3} \mathrm{PO}_{4}$-added garnettype $\mathrm{Li}_{65} \mathrm{La}_{3} \mathrm{Zr}_{15} \mathrm{Ta}_{0.5} \mathrm{O}_{12}$ for Li-dendrite suppression. Journal of Power Sources 354: 68-73.

36. Liu J, Galpaya D, Yan L, Sun M, Lin Z, et al. (2017) Exploiting a robust biopolymer network binder for an ultrahigh-areal-capacity Li-S battery. Energy Environmental Science 10(3): 750-755.

37. Kim JK (2017) Hybrid gel polymer electrolyte for high-safety lithiumsulfur batteries. Materials Letters 187: 40-43.

38. Wu N, Wang W, Wei Y, Li T (2017) Studies on the effect of nano-sized MgO in magnesium-ion conducting gel polymer electrolyte for rechargeable magnesium batteries. Energies 10(8).

39. Tripathi M, Kumar A (2018) Zinc oxide nanofiller-based composite polymer gel electrolyte for application in EDLCs. Ionics 24(10): 31553165.

40. Damen L, Hassoun J, Mastragostino M, Scrosati B (2010) Solid-state, rechargeable $\mathrm{Li} / \mathrm{LiFePO}_{4}$ polymer battery for electric vehicle application. Journal of Power Sources 195(19): 6902-6904.

41. Fu K, Gong Y, Hitz G, McOwen D, Li Y, et al. (2017) Three-dimensional bilayer garnet solid electrolyte based high energy density lithium metalsulfur batteries. Energy \& Environmental Science 10(7): 1568-1575.

42. Zhai P, Peng HJ, Zhang Q (2017) Scaled-up fabrication of porousgraphene-modified separators for high-capacity lithium-sulfur batteries. Energy Storage Materials 7: 56-63.
43. Zhu JD, Yeqian Ge, David Kim, YaoLu, Chen Chen, et al. (2016) A novel separator coated by carbon for achieving exceptional high performance lithium-sulfur batteries. Nano Energy 20: 176-184.

44. Yang D, He L, Liu Y, Yan W, Liang S, et al. (2019) An acetylene black modified gel polymer electrolyte for high-performance lithium-sulfur batteries. Journal of Materials Chemistry A 7(22): 13679-13686.

45. Song S, Lei Shi, Shiyao Lu, Yuanchao Pang, Yuankun Wang, et al. (2018) A new polysulfide blocker - poly(acrylic acid) modified separator for improved performance of lithium-sulfur battery. Journal of Membrane Science 563: 277-283.

46. Xiang Y, Wang Z, Qiu W, Guo Z, Liu D, et al. (2018) Interfacing soluble polysulfides with a $\mathrm{SnO}_{2}$ functionalized separator: An efficient approach for improving performance of Li-S battery. Journal of membrane science 563: 380-387.

47. Zhang Z, Lai Y, Zhang Z, Zhang $\mathrm{K}$, Li J (2014) $\mathrm{Al}_{2} \mathrm{O}_{3}$-coated porous separator for enhanced electrochemical performance of lithium sulfur batteries. Electrochimica Acta 129: 55-61.

48. Li J, Huang Y, Zhang S, Jia W, Wang X, et al. (2017) Decoration of silica nanoparticles on polypropylene separator for lithium-sulfur batteries. ACS applied materials interfaces 9(8): 7499-7504.

49. Liu Y, Liu Q, Xin L, Liu Y, Yang F, et al. (2017) Making Li-metal electrodes rechargeable by controlling the dendrite growth direction. Nature Energy 2(7).

50.Wang Y, Liyi S, Hualan Z, Zhuyi W (2018) Polyethylene separators modified by ultrathin hybrid films enhancing lithium ion transport performance and Li-metal anode stability. Electrochimica Acta 259: 386-394.

51. Li N, Qian Ye, Kun Zhang, Huibo Yan, Chao Shen, et al. (2019) Normalized lithium growth from the nucleation stage for dendrite-free lithium metal anodes. Angew Chem Int Ed Engl 58(50):18246-18251.

52. Liu Y, Dingchang Lin, Pak Yan Yuen, Kai Liu, Jin Xie, et al. (2017) An artificial solid electrolyte interphase with high Li-ion conductivity, mechanical strength, and flexibility for stable lithium metal anodes. Adv Mater 29(10).

53. Zhang AY, Fang X, Shen C, Liu Y, Zhou C (2016) A carbon nanofiber network for stable lithium metal anodes with high coulombic efficiency and long cycle life. Nano Research 9(11): 3428-3436.

54. Deng Y, Lu H, Cao Y, Xu B, Hong Q, et al. (2019) Multi-walled carbon nanotube interlayers with controllable thicknesses for high-capacity and long-life lithium metal anodes. Journal of Power Sources 412: 170179.

55. Liu L, Yin XY, Li JY, Guo GY, Wan LJ (2018) Ladderlike carbon nanoarrays on $3 \mathrm{D}$ conducting skeletons enable uniform lithium nucleation for stable lithium metal anodes. Chem Commun (Camb) 54(42): 5330-5333.

56. Zhang YJ, Bai W, Wang X, Xia X, Gu C, et al. (2016) In situ confocal microscopic observation on inhibiting the dendrite formation of a-CN Li electrode. Journal of Materials Chemistry A 4(40): 15597-15604.

57. Huang S, Lu Tang, Najafabadi SH, Shuo Chen, Zhifeng Ren (2017) A highly flexible semi-tubular carbon film for stable lithium metal anodes in high-performance batteries. Nano Energy 38: 504-509.

58. Liang Z, Zheng G, Liu C, Liu N, Li W, et al. (2015) Polymer nanofiberguided uniform lithium deposition for battery electrodes. Nano Letters 15(5): 2910-2916.

59. Kong LL, Wang L, Chao Ni Z, Liu S, Ran Li G, et al. (2019) Lithiummagnesium alloy as a stable anode for lithium-sulfur battery. Advanced Functional Materials 29(13).

60. Zhong H, Sang L, Ding F, Song J, Mai Y (2018) Conformation of lithiumaluminium alloy interphase-layer on lithium metal anode used for solid state batteries. Electrochimica Acta 277: 268-275. 
61. Ye H, Zheng ZJ, Yao HR, Liu SC, Zuo TT, et al. (2019) Guiding uniform Li plating/stripping through lithium-aluminum alloying medium for longlife li metal batteries. Angew Chem Int Ed Engl 58(4): 1094-1099.

62. Qiu H, Tang T, Muhammad Asif, Wei Li, Teng Zhang, et al. (2019) Stable lithium metal anode enabled by lithium metal partial alloying. Nano Energy 65: 103989.

63. Lin D, Liu Y, Liang Z, Lee HW, Sun J, et al. (2016) Layered reduced graphene oxide with nanoscale interlayer gaps as a stable host for lithium metal anodes. Nat Nanotechnol 11(7): 626-632.
64. Liu Y, Lin D, Liang Z, Zhao J, Yan K, et al. (2016) Lithium-coated polymeric matrix as a minimum volume-change and dendrite-free lithium metal anode. Nat Commun 7: 10992.

65. Li Q Zhu SP, Lu YY (2017) 3D porous Cu current collector/Li-metal composite anode for stable lithium-metal batteries. Advanced Functional Materials 27(18).

66. Yang CP, Yin YX, Zhang SF, Li NW, Guo YG (2015) Accommodating lithium into 3D current collectors with a submicron skeleton towards long-life lithium metal anodes. Nat Commun 6: 8058. 